\title{
Silver ions as EM marker of congo red ligation sites in amyloids and amyloid-like aggregates
}

\author{
Janina Rybarska', Leszek Konieczny', Anna Jagusiak'1, Katarzyna Chłopaś', \\ Grzegorz Zemanek1, Barbara Piekarska1, Barbara Stopa', Piotr Piwowar², \\ Olga Woźnicka ${ }^{3}$ and Irena Roterman ${ }^{4 \bowtie}$
} ${ }^{1}$ Chair of Medical Biochemistry, Medical College - Jagiellonian University, Kraków, Poland; 2 Department of Measurements and Electronics, AGH
University of Science and Technology, Kraków, Poland; ${ }^{3}$ Department of Cell Biology Imaging, Faculty of Biology, Earth Sciences, Jagiellonian University, Kraków, Poland; “Department of Bioinformatics and Telemedicine, Medical College - Jagiellonian University, Kraków, Poland

\begin{abstract}
Congo red (CR) is a known selective amyloid ligand. The focus of our work is identification (by EM imaging) of dye binding sites and their distribution in amyloids and amyloid-like aggregates formed in vitro. In order to produce the required contrast, CR has been indirectly combined with metal via including Titan yellow (TY) by intercalation which exhibits a relatively strong affinity for silver ions. The resulting combined ligand retains its ability to bind to proteins (which it owes to CR) and can easily be detected in EM studies thanks to TY. We have found, however, that in protein aggregates where unfolding is stabilized by aggregation and therefore is irreversible, TY alone may serve as both, the ligand and the metal carrier. The formation of ordered structures in amyloids was studied using IgG light chains with amyloidogenic properties, converted into amyloids by shaking. The resulting EM images were subjected to interpretation on the basis of the authors' earlier research on the CR/light chain complexation process. Our results indicate that dimeric light chains, which are the subject of our study, produce amyloids or amyloid-like complexes with chainlike properties and strong helicalization tendencies. Cursory analysis suggests that the edge polypeptide loops belonging to unstable light chains form intermolecular bridges which promote creation of loose gel deposits, or are otherwise engaged in the swapping processes leading to higher structural ordering.
\end{abstract}

Key words: Congo red, Titan yellow, amyloids, supramolecular dyes, metal markers, light chain, amyloid-like aggregates, edge loop

Received: 17 July, 2016; revised: 16 August, 2016; accepted: 05 September, 2016; available on-line: 19 December, 2016

$\square$ e-mail: myroterm@cyf-kr.edu.pl

Abbreviations: CR, Congo red; TY, Titan yellow; EM, electron microscope; FOD model, fuzzy oil drop model

\section{INTRODUCTION}

Amyloids are protein aggregates which resist biological degradation mechanisms due to their structural ordering. The formation of such structures (often referred to as amyloidogenesis) has been the subject of numerous studies (Dealwis, 2004; Barrow et al., 1992; Kelly, 1997; Bross et al., 1999; Teng et al., 2012; Pellarin et al., 2010). Amyloid deposits are formed from unstable, partly unfolded proteins, but aggregation usually results in tight packing and the resulting structures become impervious to natural degradation. As can be expected, amyloid aggregates are chainlike struc- tures consisting of interlinked proteins, which - by way of successive transformations - produce higher-order units. In vivo, amyloids form slowly and, much like crystals, exhibit a high degree of structural ordering, which is revealed by birefringence in polarized light. In contrast, amyloids (or amyloid-like aggregates) rapidly synthesized under laboratory conditions exhibit variable levels of ordering. Despite these differences, experimental work provides much insight into the intermediate stages of protein aggregation. Amyloidogenesis remains a complex issue and there is no consensus regarding its course and the overall structure of its products. Some of the observed insoluble aggregates do not qualify as amyloids under the generally accepted criteria, since they do not attain sufficient structural ordering. It seems that this issue merits further attention (Fink, 1998; Khurana et al., 2001; Helms et al., 1996; Maji et al., 2009).

As amyloids are not classic crystals and do not lend themselves to crystallographic analysis, EM remains the method of choice for studying their properties. However, applying EM in molecular research requires a suitable contrast and staining which can reveal the location of specific target structures. The study presented here relies on silver ions, introduced into an amyloid-specific ligand (CR) in order to reveal the distribution of sites binding the dye in the in vitro produced amyloid-like aggregates. $\mathrm{CR}$ is doped with silver in an indirect manner, i.e. by including TY which in turn binds $\mathrm{Ag}^{+}$ions. The structure and properties of TY resemble those of CR; hence TY can either be used on its own or introduced into micellar CR through intercalation, yielding complex ligands. The work presented here is a continuation of our earlier research on labeling amyloid-specific ligands with metal ions (Chłopaś et al., 2015).

Amyloid-like aggregates are obtained through shaking - a process which exploits the amyloidogenic propertis of IgG light chains isolated from urine of patients suffering from multiple myeloma. Immunoglobulin light chain L Lambda dimers were used in this work as a material with well-known structural properties and amyloidogenic tendencies.

Conclusions from the analysis of ion-stained amyloid-like aggregates under electron microscopy are presented in the Results and the Discussion sections.

\section{MATERIALS AND METHODS}

Reagents. CR with dye content of 97\% was purchased from Aldrich Chemical Company Inc. It was used without further purification. TY was obtained from 
BDH Chemicals Ltd - England. The true content of the silver binding dye in the commercial material was measured by titration with $\mathrm{AgNO}_{3}$ at $407 \mathrm{~nm}$ based on TY: $\mathrm{Ag}^{+}$1:1 molar ratio (Chłopaś et al., 2015). The presence of some inactive impurities was deemed to have no impact on the staining procedure.

Other reagents used were of analytical grade.

Electron Microscopy. Transmission electron micrographs were collected using the JEOL 2100 HT microscope. Typical nominal magnifications ranged from 30-75000. Stained samples were deposited on Formvar 300-Mesh copper grids for analysis. Aggregates were analyzed without using negative staining. The introduced dye contrast was solely of silver origin.

Light microscopy. A Carl Zeiss Jena light microscope with polarization and fluorescence equipment was used for initial analyses preceding EM analysis.

Dynamic light scattering. LS - Dyna-Pro800 produced by Protein Solutions INC, with a measuring range of $0.5-50 \mathrm{~nm}$ was used to verify the supramolecular nature of CR and TY dyes.

Other laboratory equipment. Standard laboratory equipment was used to complete experimental studies.

Proteins. Immunoglobulin light chains were obtained from the urine of myeloma patients. They were salted out and, following intensive dialysis, further purified by molecular gel filtration.

Amyloid and amyloid-like aggregates were prepared by shaking at $40 \mathrm{C} \operatorname{deg}$ in $0.05 \mathrm{M}$ acetate buffer, $\mathrm{pH} 5.2$, or $0.05 \mathrm{M}$ glycine $/ \mathrm{HNO}_{3}$ buffer, $\mathrm{pH} 2.15$, using protein concentrations of $8-10 \mathrm{mg} / \mathrm{ml}$. Aggregation was performed with or without dithiotreitol added at a concentration of $0.6 \mathrm{mg} / \mathrm{ml}$.

The precipitate was washed with $0.3 \% \mathrm{KNO}_{3}$ or by $0.05 \mathrm{M}$ Tris $/ \mathrm{HNO}_{3}$ buffer, $\mathrm{pH}=8.2$. In selected cases, a $0.05 \mathrm{M}$ acetate buffer, $\mathrm{pH}=5.2$, was used instead.

Light chains. The L Lambda light chains used in this work adopt a dimeric form. They originate from different patients and thus differ in terms of stability. This factor was evaluated on the basis of CR binding susceptibility, since CR is known to form complexes by penetrating into the destabilized protein domains. Complexation occurs at room temperature or may require suitable heating. The stability of $\mathrm{V}$ domains is greater in dimers due to interface interaction of the $V$ domains. The purity of $\mathrm{L}$ chains was evaluated by analysis using agarose and polyacrilamide gel electrophoresis (without SDS) performed in a $0.06 \mathrm{M}$ veronal buffer $(\mathrm{pH}=8.6)$ or $0.05 \mathrm{M}$ Tris buffer $(\mathrm{pH}=8.2)$.

Fibril formation by Beta-amyloid peptides. $0.5 \mathrm{mg}$ of amyloid Beta-peptides was solubilized in $100 \mu \mathrm{l} \mathrm{H}_{2} \mathrm{O}$. Subsequently, $30 \mu \mathrm{l}$ of this solution was mixed with $30 \mu \mathrm{l}$ of $0.5 \mathrm{M}$ Tris $/ \mathrm{HNO}_{3}$ buffer $(\mathrm{pH}=7.35)$ and incubated over four days at $37 \mathrm{C}$.

Staining of $\mathbf{L}$ chain (immunoglobulin) aggregates. The silver-labeled stain for EM studies was prepared by forming the combined micellar structures composed of $\mathrm{TY}, \mathrm{Ag}^{+}$and $\mathrm{CR}$, mixed at molar ratios of 1:1:1 or 1:1:0.5 respectively, with initial concentrations of TY equal to $4 \mathrm{mg} / \mathrm{ml}$.

To obtain a proper dye combination, the mixture of TY and CR was heated for $20 \mathrm{~min}$ at $60^{\circ} \mathrm{C}$ and then cooled slowly to room temperature. Silver ions $\left(\mathrm{AgNO}_{3}\right)$ were added with stirring to form the complex. The concentration of dyes used for staining of protein precipitates was generally at the level of $2 \mathrm{mg}$ of each dye per $\mathrm{ml}$. The silver-containing stains (TY) $\mathrm{Ag}^{+} / \mathrm{CR}$ or $\mathrm{TY} / \mathrm{Ag}^{+}$) were added to the aggregates and incubated for $30 \mathrm{~min}$. The stained aggregates were then washed with $0.025 \mathrm{M}$ Tris $/ \mathrm{HNO}_{3}$ buffer $(\mathrm{pH}=8.2)$ or, in some experiments, with $0.025 \mathrm{M}$ acetate buffer $(\mathrm{pH}=5.2)$. This was done to verify the stability of the complex at different $\mathrm{pH}$ levels. The stained precipitate was suspended in water directly prior to the EM analysis. Silver-containing stains were kept in the dark before use.

To verify that silver added to dyes forms part of the complex, the stain and free silver ions were separated by agarose electrophoresis using $0.05 \mathrm{M}$ Tris/ $\mathrm{HNO}_{3}$ buffer $(\mathrm{pH}=8.2)$ in order to verify that the silver ions migrate to the anode (together with dyes) and not to the cathode (as free ions). To localize the silver in the plate, agarose plates are placed in a solution of sodium dithionite to discolor CR spots and simultaneously reduce silver ions (Stopa et al. 2003). Reduction continues until CR spots disappear. TY is then transferred to the filter paper by blotting. Insoluble silver (if present) remains in the agarose as black remnants of dye spots. The control sample $\left(\mathrm{AgNO}_{3}\right)$ and the excess of silver ions in stained samples manifest as spots migrating toward the cathode. The reduction-mediated blackening of stained aggregates may also be observed under a light microscope.

Models of aggregates. Hypothetical models of aggregates were constructed on the basis of former studies regarding CR location in the $\mathrm{V}$ domains of immunoglobulin light chains (Stopa et al., 2003), as well as current experimental results.

\section{RESULTS}

\section{Silver-carrying EM stain for amyloids}

The formation of ordered structures in the amyloidogenesis process protects peptide bonds from natural degradation. Such degradation is further inhibited by the tight packing of proteins in amyloid plaques. As a rule, amyloids consist of partly unfolded proteins. This means that parts of the protein molecule are not involved in the unfolding process. The aggregation mechanism is hence more specifically targeted and the final product is expected to be ordered rather than amorphous (Jiang et al., 2001; Mizobata et al., 1994; Quintas et al., 2001; Colon et al., 1992).

As can be expected, amyloid aggregates are chain-like structures which consist of protein monomers wound into higher-order structures. In this sense they resemble peptide chains; however instead of amino acids their building blocks are entire protein molecules.

Understanding of this problem enables us to study the onset and further progress of amyloid aggregation (Quintas et al., 2001; Colon et al., 1992). Our work is based on studying this process using electron microscopy (EM) as the principal analytical tool.

We trace the presence of amyloid aggregates using Congo red (CR) - a well-known selective amyloid ligand which helps to reveal the presence of its binding sites and therefore facilitate molecular studies of amyloids (Quintas et al., 2001; Colon et al., 1992; Inouye et al., 2000).

In order to achieve a suitable contrast for EM, the ligands have been doped with silver ions; however the metal is not directly bound to CR (which has poor complexation properties) - instead CR is intercalated as a complex with another, similar substance which can bind silver ions and readily associates with CR - Titan Yellow (TY) (King et al., 1967) (Fig. 1, Fig. 2). The supramolecular nature of both dyes and their ability to form 


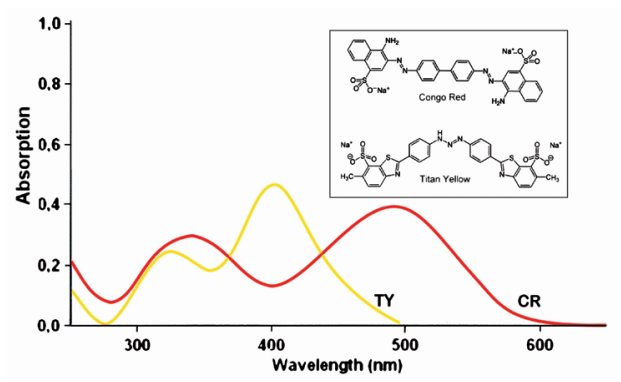

Figure 1. CR and TY - formulas and spectra.
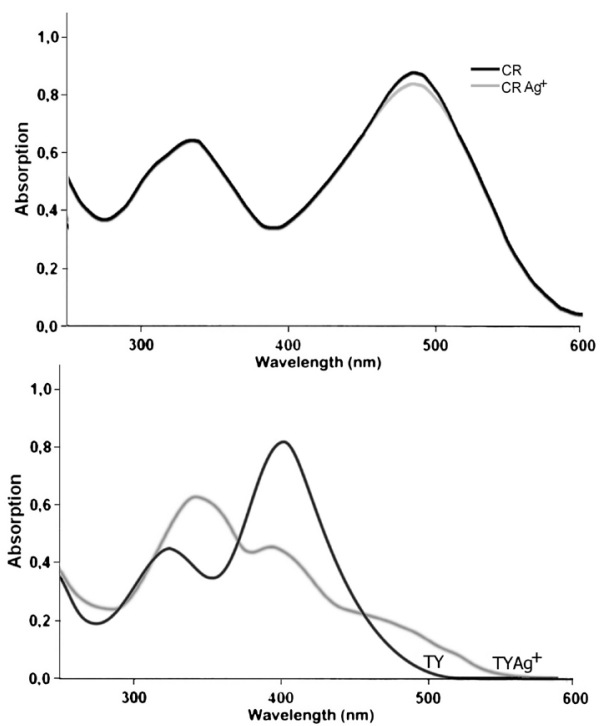

Figure 2. Different effects of $\mathrm{Ag}^{+}$ion complexation on spectral properties of the dyes - negligible for CR and significant for TY.

combined micellar structures (Fig. 3) promote mutual association (Fig. 4).

TY's ability to bind silver ions is due to its centrally located tri-azene group, attracting one ion per dye molecule (Chłopaś et al., 2015). The supramolecular ligand binds to proteins as a package consisting of several selfassembled molecules, resulting in local concentration of silver nanoparticles, recognizable under EM.

The $\mathrm{TY} / \mathrm{Ag}^{+}$binding strength is verified by testing the stability of the complex in the presence of anions

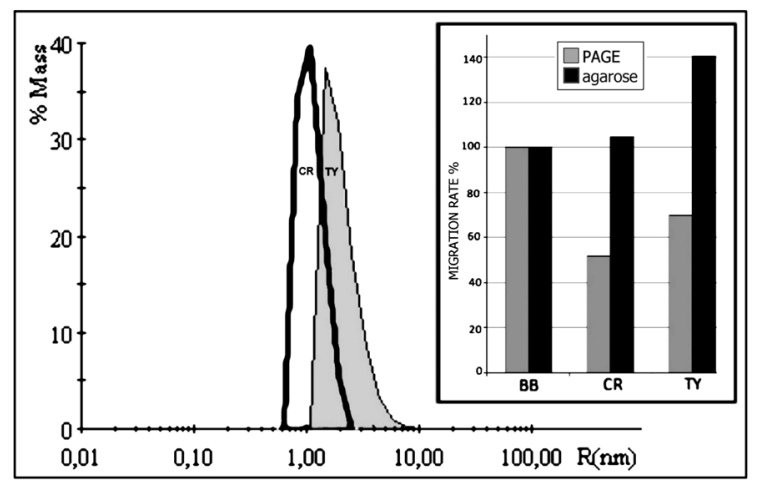

Figure 3. Supramolecular character of CR and TY evidenced by the DLS analysis (white and gray respectively) and the relative electrophoretic migration rate in a molecular sieve system $(15 \%$ polyacrylamide gel) versus agarose (1\%); both compared to the migration rate of a bromophenol blue dye, which is assumed to be $100 \%$ (see inset).

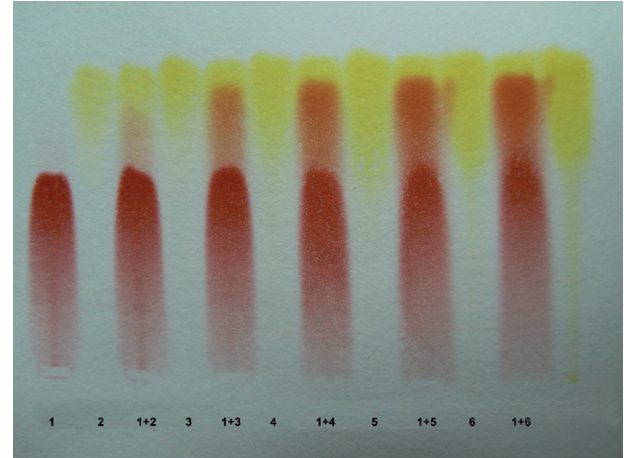

Figure 4. Mutual association of CR (1) and TY $(2,3,4,5,6)$ evidenced by increasing participation of CR in the fast-migrating fraction in electrophoresis (caused by increasing amounts of TY added to an excess of $(R)$.

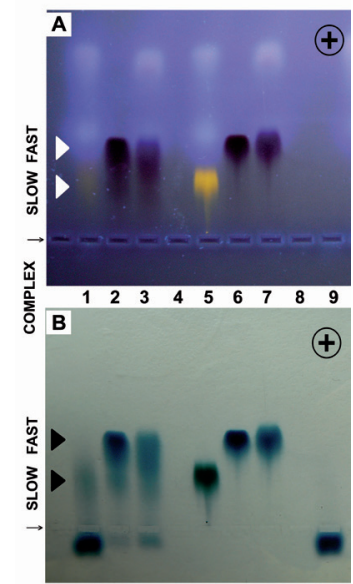

Figure 5. Heat-induced formation of CR and TY complexes with the L lambda chain.

(A) agarose electrophoresis - registration of dyes under UV. (B) the same electrophoretic plate following reduction with sodium dithionite to discolor CR spots, which are then stained with the bromophenol blue dye to reveal proteins. 1, L lambda + TY; 2 , L Lambda + CR; 3, L lambda + CR/TY; 1,2,3-40 4 C deg - 10 min; 5, L lambda + TY; 6, L lambda + CR; 7, L Lambda + CR/TY; 5,6,7 - 60 ${ }^{\circ} \mathrm{C}$ deg - 10 min; 9, L chain

which, when mixed with silver ions, form poorly soluble salts with a known solubility product constant. It was found that the TY/ $\mathrm{Ag}^{+}$complex forfeits its silver ion to iodates and thiols, but in the presence of Tris as an additional silver complexation component it survives chlorides even at a concentration of $0.15 \mathrm{M}$. Electrophoretic migration of the $\mathrm{TY} / \mathrm{Ag}^{+}$complex in $0.05 \mathrm{M}$ Tris/ $\mathrm{HNO}_{3}$ buffer $(\mathrm{pH}=8.2)$ in the direction of the anode proves its stability under these conditions. It was found that the use of an acetate buffer $(0.05 \mathrm{M} ; \mathrm{pH}=5.2)$ is also possible although the resulting complex is less stable.

The tri-azene bridge, centrally located in the TY molecule, seems essential for complexation of silver ions. Direct Yellow 28 (DY28), a similar dye, lacks such a bond and does not form stable complexes with $\mathrm{Ag}^{+}$.

The presence of complexed silver ions in the experimental sample can be easily determined by reduction with sodium dithionite, with the resultant darkening of samples serving as a direct evidence of the presence of silver.

TY is a supramolecular system with a ribbon-like structure, commonly assumed by symmetrical, planar molecules which exhibit polarity in their distal fragments and contain a nonpolar central fragment composed of 


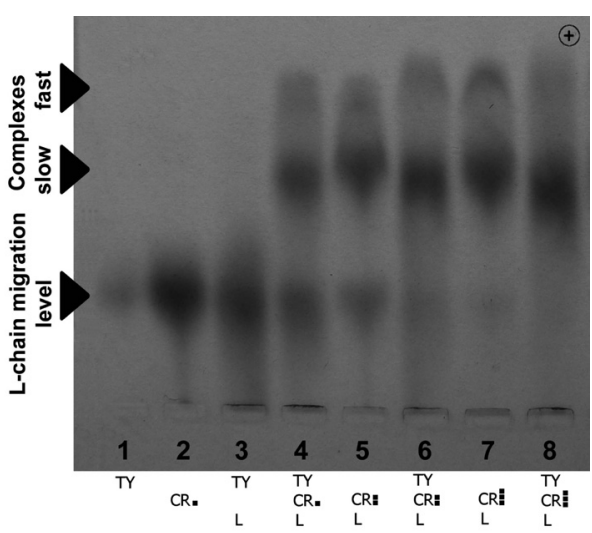

Figure 6. Evidence of the CR drawing effect necessary for the engagement of TY in the $L$ chain complexation - seen as the increased effectiveness of formation of complexes with TY contribution, resulting in increased involvement of CR (arrows).

aromatic rings (Fig. 1). Unlike CR, TY does not readily penetrate into the protein complexes. The formation of a complex requires irreversible aggregation-stabilized unfolding of the protein molecule - here, TY is outcompeted for access to the binding site by the trans-located polypeptide loop present in the reversible dynamic structure of the protein (Fig. 5). The polypeptide loop effectively occupies the binding locus in preference to TY (but not to CR) and causes removal of the dye from the complex.

Fortunately, the penetration abilities of TY are substantially improved when it forms a co-micellar structure with CR (Fig. 6) - although some aggregates which depend upon intrinsically stabilized unfolding of proteins may be tagged by TY alone in the EM studies.

\section{EM visualization of dye-silver-stained amyloid-like aggregates}

Loose strips of gel formed in vitro by $\operatorname{IgG}$ light chains, observed at the edges of more densely packed structures (where the gel is less dense, allowing penetration), easily bind the $\mathrm{TY} / \mathrm{Ag}^{+}$complex, revealing the molecular structure of aggregates. The silver-doped ligand can be detected as clusters of granularities, reflecting the distribution of dye particles in the gel (Fig. 7).

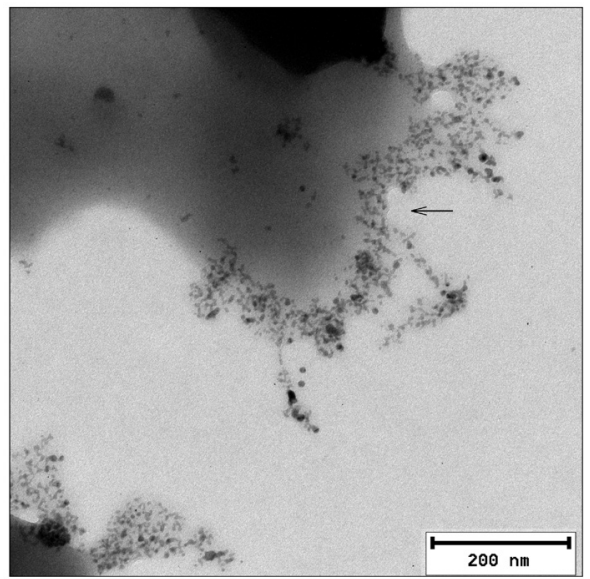

Figure 7. Edge of an amyloid particle with reduced compactness, penetrated and contrasted by $\mathrm{TY} / \mathrm{Ag}^{+}$(seen under EM). Some spiral organization within the amyloid-like material is locally noticeable (see arrow).

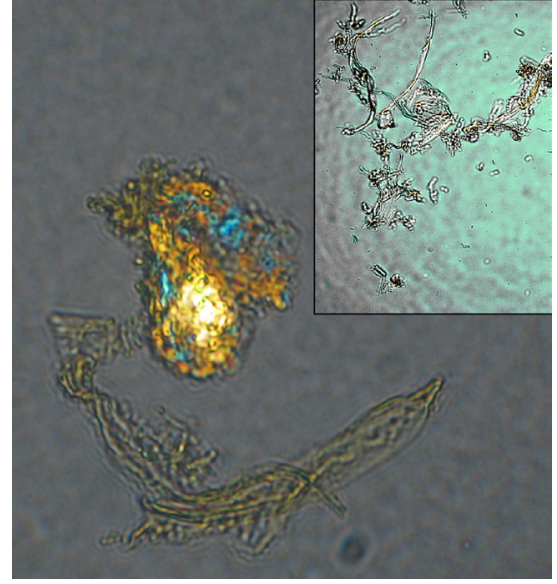

Figure 8. Local para-crystalline organization present in the amyloid-like material revealed by active rotation of polarized light. Inset: fibrillar nature of aggregates.

According to the common definition, an amyloid is a protein aggregate with a fibrillar structure which binds CR and exhibits birefringence in polarized light (Howie et al., 2009; Picken 2001). Amyloid-like gels formed by shaking at $45-50^{\circ} \mathrm{C}$ deg manifest themselves under light microscope imaging as veils, often lacking birefringence (although they retain their ability to bind $\mathrm{CR}$ ). When the temperature drops below $35^{\circ} \mathrm{C}$ (and then further below $30^{\circ} \mathrm{C}$ ) birefringent structures become more prevalent and many fibrillar structures begin to emerge (Fig. 8).

EM imaging reveals loosely packed amyloid-like structures as short chains of aggregates randomly strewn across the field of vision. Some of the longer chains are positioned in parallel with respect to one another, or form spirals (Fig. 9 - arrows). This suggests a degree of ordering which, in turn, may provide a starting point for the onset of birefringence. A typical higher-order structure is a circular shape comprising tightly wound spiral chains of aggregates (Fig. 10, $10 \mathrm{~A})$. The observed threadlike structures are formed from loosely bound grains of contrast. These can be assumed to correspond to light-chain molecules interlinked by exposed polypeptide loops. On the other hand, EM imaging also reveals other types of ordered

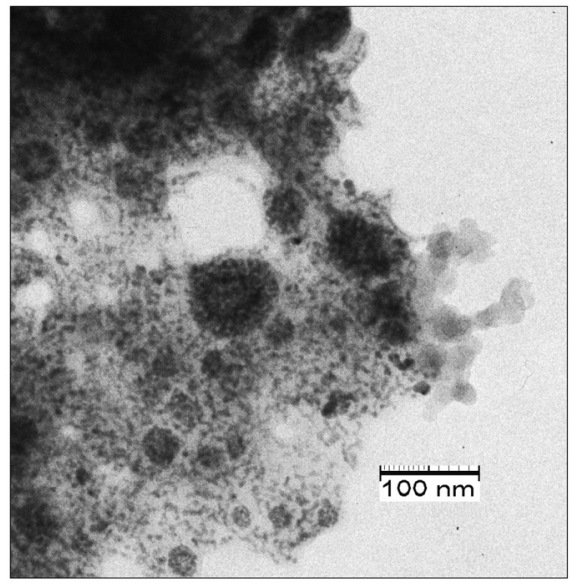

Figure 9. A piece of amyloid-like material stained by CR/TY/ $\mathrm{Ag}^{+}$supramolecular dyes. Randomly distributed short protein threads are marked by contrast, with a noticeable tendency to form spirals. 


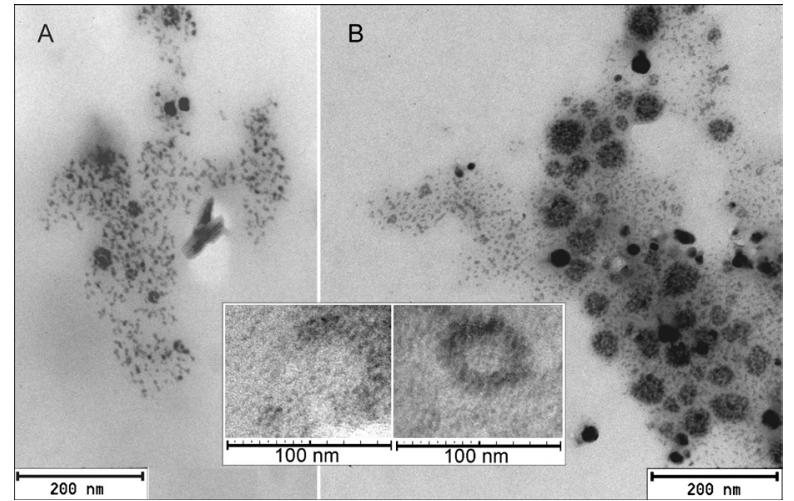

Figure 10. Amyloid-like particles seen under EM.

(A) mostly random distribution of short, contrasted aggregate chains. (B) intensive accumulation of ordered spiral forms. Inset: spiral amyloid aggregates undergoing organization.

structures which are not spiral and do not bind CR (or which bind it poorly). Such structures may emerge as a result of combining light-chain molecules by swapping, enabling protein domains to revert to their fully packed form (Bennet et al., 1995; Cohen 1999; Nelson et al., 2006; Nagradova 2002).

\section{Structural interpretation of dye silver stain binding by the $\mathrm{L}$ chain $\mathrm{V}$ domain}

In an attempt to explain the observed aggregation mechanism at the molecular level, we refer to the $\mathrm{V}$ domain structure of the $\operatorname{IgG}$ light chain. This domain comprises two $\beta$-sheets linked by a disulfide bond, usually referred to as the upper core and the lower core, respectively (Fan et al., 1992; Ewert et al., 2004) (Fig. 11). Loops which form the lower core are additionally stabilized in a dimeric form via interactions with the $\mathrm{V}$ domain of the complementary chain. Amyloid aggregation is therefore mostly due to polypeptide chains forming the upper core. The relative instability of the upper core is also due to the presence of the $\mathrm{N}$-terminal fragment which, being a terminal fragment, is unstable by nature and easily displaced from its packing locus. As has been revealed in our study, displacement of the N-terminal fragment creates a cavity which the supramolecular CR ligand ea-

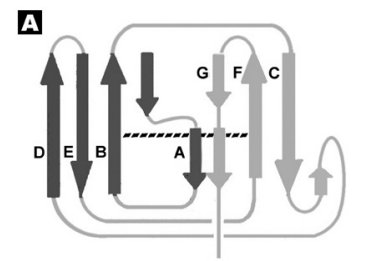

a

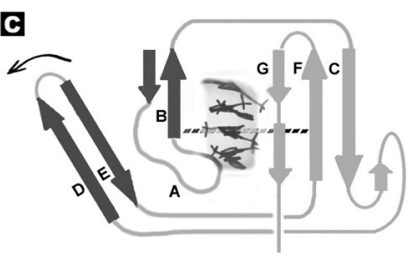

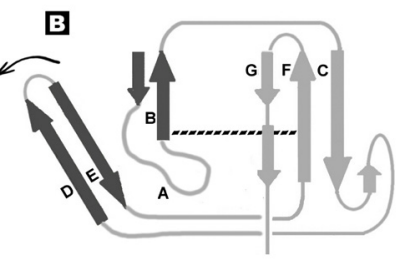

D

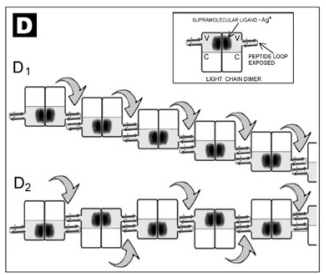

Figure 11. Hypothetical mechanism of the $L$ chain/V domain involvement in aggregation.

(A, B, D) schematic depiction of formation of the observed chainlike aggregates and spiral structures. (C) location of the 4-molecule CR ligand in the V domain, along with the displaced N-terminal chain fragment. gerly occupies, preventing the domain from reverting to its initial, native form (Piekarska et al., 2001).

Figure 11 shows a schematic diagram of the $\mathrm{V}$ domain with $\beta$ twists forming the upper core represented by black arrows. Displacement of the N-terminal fragment from its native locus (e.g. under heightened temperature) destabilizes the upper core, strongly affecting the edge loop (D-E) which may hence protrude from the domain (Yang et al., 2003; Sinha et al., 2001). As expected, mutual association of the exposed D-E loops and formation of $\beta$-bridges produces a chainlike aggregate (Fig. 11D). The resultant link comprises two D-E loops, i.e. four polypeptide chains, with preserved $\beta$ conformation.

The involvement of the $\mathrm{N}$-terminal fragment in the $\beta$-bridge is unclear as its participation would seemingly promote the formation of a six-chain bridge.

Displacement of the polypeptide loops and their involvement in aggregation creates gaps, which are further occupied by supramolecular ligands (Fig. 11) (Piekarska et al., 2001; Jagusiak et al., 2014; Król et al., 2005). The dyes penetrate and bind in between $\beta$-structural polypeptide chains (Roterman et al., 1998). Our previous studies confirm that CR attaches to the $\mathrm{V}$ domain of the light chain. The four-molecule dye ligand engages the domain once the $\mathrm{N}$-terminal fragment has been displaced. The resulting complex is found to be slow-moving in electrophoretic studies and will be referred to as such. Under favorable conditions (higher temperature; higher CR concentration) four additional CR molecules may attach to the complex, resulting in a fast-moving fraction (Fig. 5; Fig. 6) (Piekarska et al., 2001; Jagusiak et al., 2014). This complexation progresses in a stepwise fashion and with limited efficiency as it requires displacement of the C-D and E-F loops, which is more troublesome than in the case of the $\mathrm{N}$-terminal fragment. The overall process produces a streak-like pattern on

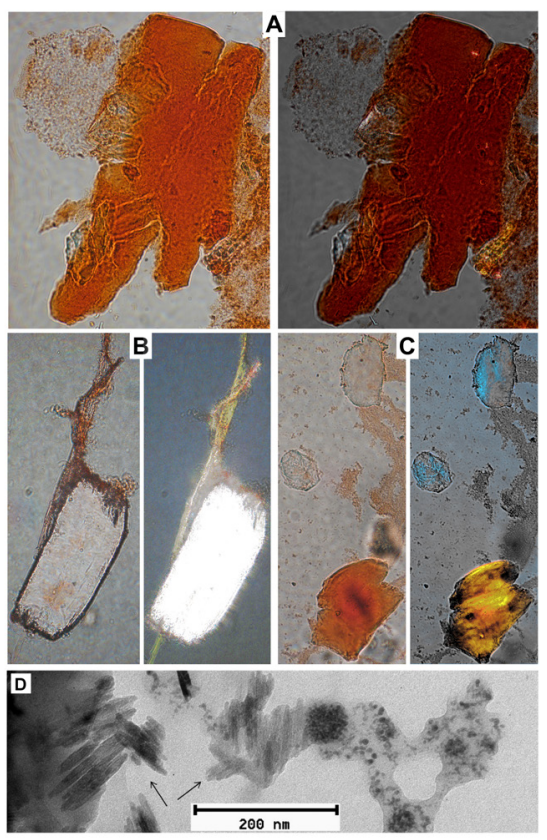

Figure 12. Microscopic images presenting:

(A, B, C) selected examples of combined CR-binding and nonbinding structures formed in the presence of CR (light microscope). Left - visible light, right - polarized light. (D) amyloid-like aggregate particle involving structural forms with high and low CR binding affinity (arrows). 


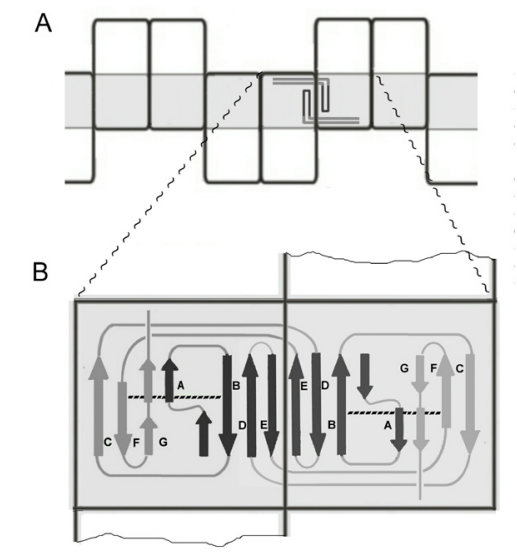

Figure 13. Schematic depiction of the $\mathrm{V}$ domain association based on the swapping mechanism - formation of cross-Beta structures (A). Same structure with more details (B).

the electrophoretic plate. It seems that the complex may bind ligands consisting of five, six, seven or eight dye molecules (Piekarska et al., 2001; Jagusiak et al., 2014; Król et al., 2005).

\section{Swapping as the hypothetical mechanism of the ordered $\mathrm{L}$ chain aggregation}

Aggregates formed by swapping may prove unsuitable for binding supramolecular ligands in their basic form, since their domains are often fully packed. This conclusion is supported by detection of nonbinding or weakly binding CR amyloid-like structures in the aggregation products (Fig. 12) (Wood et al., 1996; Heegaard et al., 2000; Liu et al., 1998). The coupling of $\mathrm{V}$ domain chains by swapping is illustrated in Fig. 13.

The relative structural freedom of amyloid-like aggregates and their variable binding mechanisms (formation of bridges and/or swapping) indicate that the phenomenon is quite complex and requires an in-depth analysis. Additionally, the means by which aggregates bind the CR molecules is unclear and has been the focus of varying interpretations (Frid et al., 2007; Turnell et al., 1992; Jin et al., 2003; Elhaddaoui et al., 1995; Klunk et al., 1999; $\mathrm{Kim}$ et al., 2003). The proposed use of metal ions as a contrast carried by protein ligands may shed more light on this important matter.

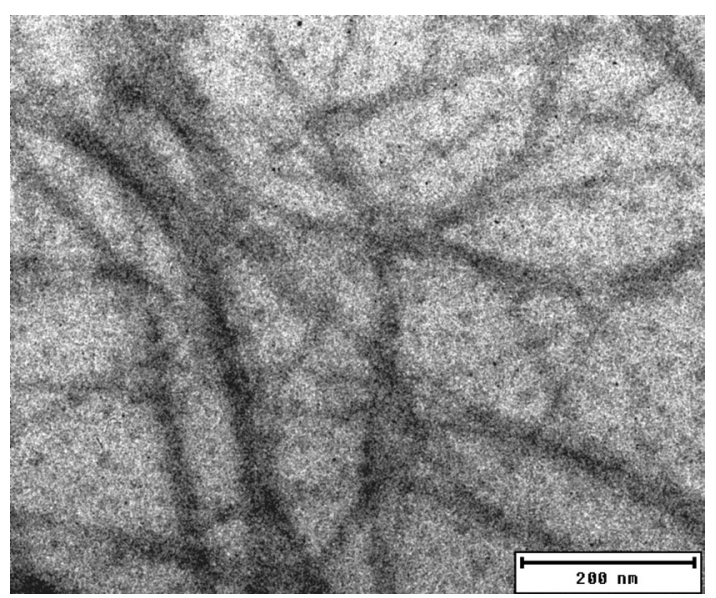

Figure14. Amyloid-derived fibrils stained by CR carrying TY and silver ions, reduced (partly) under EM.
Dye-silver staining of amyloid $\beta$-peptide-derived fibrils

Figure 14 presents the use of dye-silver staining (CR, TY, $\mathrm{Ag}^{+}$) to reveal the standard dye-amyloid complex in the case of amyloid $\beta$-peptide derived fibrils (Wu et al., 2012; Lu et al., 2013; Serpel, 2000; Tycko, 2004). Periodic distribution of contrast density along fibrils suggests a twisted ribbon structure (Pedersen et al., 2010; Petkova et al., 2006; Bose et al., 2010; Lendel et al., 2010; Lührs et al., 2005). The formation of amyloids from peptides differs from the previously discussed light-chain aggregation process as there is no protein ballast; however the underlying self-organization mechanism and the resulting structure appear to be similar.

The periodicity observed in fibrils suggests a twisted ribbon-like nature of amyloid threads (Petkova et al., 2006; Bose et al., 2010; Lendel et al., 2010; Lührs et al., 2005; Skowronek et al., 1998). Coating of fibrils by silver may be interpreted as adhesion of the supramolecular dye (carrying silver ions) to the amyloid fibril ribbon. Contrast is provided by precipitated silver following reduction of silver ions under EM, revealing the structural characteristics discussed above.

\section{DISCUSSION}

In our research, we have attempted to analyze the formation of ordered structures in the process of amyloidogenesis based on the distribution of specific amyloid ligands which have been imbued with contrast properties by intercalation of $\mathrm{Ag}^{+}$ions. Our ligands include supramolecular dyes - CR and/or TY (Skowronek et al., 1998; Iconomidou et al., 2003; Stopa et al., 1998). Both dyes, when mixed together, are capable of selective binding to amyloid-like aggregates and the distribution of such combined ligands can be studied under EM thanks to the silver's contrast properties.

Metal ion complexes have many applications in diagnostics and therapy. Complexation of metal ions increases their solubility while also reduces their toxicity. In this work, however, silver ions are used for an entirely different purpose: to reveal the distribution of Congo red in amyloid aggregates under electron microscopy, even though the dye itself does not readily form complexes with this metal. In order to overcome this difficulty, we have created a supramolecular ligand consisting of two distinct organic compounds: Congo red and Titan yellow, the latter of which exhibits strong affinity for silver ions. Both dyes must combine to form a ligand, but the presence of Congo red is arguably more important since it is a well-known amyloid ligand and its distribution is the principal focus of EM analysis. The silver-binding component (TY) should not appreciably alter the supramolecular structure of Congo red and should not interfere with its ability to bind to proteins.

TY appears to fulfill the above conditions. The TY molecule is structurally similar to Congo red and it forms similar supramolecular structures (although its complexes with proteins appear to be less stable in the absence of Congo red). Both dyes readily form combined micellar structures. It appears that combination of both dyes does not alter the specific complexation profile of Congo red and the aggregate supramolecular dye represents a useful means of revealing the structure of amyloids under electron microscopy.

Even though CR is widely known for its selective affinity for amyloids, its binding mechanism has not been unequivocally elucidated (Wu et al., 2007; Demeule et al., 2007; Khurana et al., 2001; Pollack et al., 1995). Controversy surrounds the means of complexation of individual 
CR molecules which bind to $\beta$-pleated sheets, as well as the penetration of self-assembled molecules as protein ligands into unstable $\beta$ areas (Wu et al., 2007; Demeule et al., 2007; Khurana et al., 2001; Pollack et al., 1995). Adhesion appears to play an important role in this process, since - in the presence of water - CR forms long ribbon-like chains with large nonpolar surfaces, shaped by associated aromatic rings of the central part of the dye molecule (Woodcock et al., 1995). Ultimately, however, the specific mechanisms of amyloid formation and Congo red binding remain unclear and continue to attract the researchers' attention (Barrow et al., 1992; Kelly 1997; Jiang et al., 2001; Qin et al., 2007).

In the study presented here, amyloid and amyloid-like structures were obtained from IgG light chains via shaking. Light chains were selected as the study subject due to their well-known structural properties, ability to bind CR and overall clinical importance (Spólnik et al., 2004). Standard amyloid fibrils were obtained using commercial $1-40$ amyloid $\beta$-peptides.

Displacement of polypeptide chain fragments in the unstable $\mathrm{V}$ domain creates suitable conditions for supramolecular ligands to penetrate in between polypeptide chains of $\beta$-pleated sheets where they can anchor themselves with their nonpolar fragments, while the distal polar groups protrude outside the protein (Roterman et al., 1998).

Increased temperature destabilizes the $\mathrm{IgG}$ light chains which form two types of complexes with supramolecular CR ligands. Such complexes are characterized by variable migration speed under electrophoresis and comprise ligands with either 4 or 5-8 dye molecules. These fractions are referred to as "slow-moving" and "fast-moving” respectively (Fig. 5; Fig. 6) (Piekarska et al., 2001). The slow-moving complex forms easily through displacement of the $\mathrm{N}$-terminal fragment from its packing locus, which is instead occupied by a 4-molecule CR ligand. Formation of the fast-moving complex occurs with greater difficulty (Piekarska et al., 2001) and only when $\mathrm{CR}$ is used as the ligand. TY alone seems to be unable to form such complexes; however, the slow-moving complex may involve TY without any participation of $\mathrm{CR}$, but only under unfolding conditions which induce oligomerization (60 deg C; 10 min - see Fig. 5), ensuring permanent displacement of the domain loops. The complexes formed by slightly destabilized $\operatorname{IgG}$ light chains and CR/TY ligands are soluble and do not - by themselves - form aggregates. Instead, aggregation requires further destabilization and sufficient exposure of polypeptide loops, which can be achieved by shaking and/or reduction of disulfide bonds. Unstable domains become susceptible to penetration by supramolecular ligands.

Reduction with sodium dithionite reveals the presence of silver (via darkening), both in the electrophoretic spots and in the TY-stained deposits. This serves as proof that silver ions are present in the sample.

Somewhat unexpectedly, microscopic imaging of amyloid-like aggregates reveals aggregates which do not bind CR (or bind it poorly) but retain birefringence under polarized light, suggesting a high degree of ordering. This phenomenon may be explained by assuming that complexation of light chain molecules occurs by way of inter-molecular swapping of edge loops (D-E). Such aggregation may produce densely packed polypeptide clusters which do not offer suitable cavities for CR ligands (Fig. 13) The structural freedom and elasticity of the resulting aggregates is limited by their high degree of structural ordering. Tight spiral winding of chains is therefore very difficult (or outright impossible). Nevertheless, un- der favorable conditions (destabilization), the aggregates remain capable of interaction with CR (Churukian, 2000; Lakdawala et al., 2002). This is due to the fact that not all fragments of the target polypeptide chain are equally stable (Tycko, 2004), providing attachment points for dyes even in aggregates formed by swapping - especially under unfolding conditions, as observed in staining studies. CR may also bind to proteins by means of adhesion due to its ribbon-like supramolecular structure presenting a large non-polar surface which may attach to fibrillar protein aggregates.

The instability of myeloma-derived light chains presents an important study subject due to their propensity to form amyloid deposits in kidneys (Schormann et al., 1995; del Pozo et al., 2008; Hurle et al., 1994; Wall et al., 1999). Assessing the degree of such instability and prediction of danger is possible on the basis of the socalled fuzzy oil drop (FOD) model (Banach et al., 2014), which determines the involvement of polypeptide chain fragment in the formation of an ordered, polar protein. The FOD model expresses the degree of participation of individual polypeptide chain fragments (especially secondary folds) in a common hydrophobic core structure. As a result, the FOD model can be used to compute the relative stability of each fragment.

Accurate prediction of amyloidogenesis would be of fundamental importance in clinical practice. Nevertheless, the problem remains a challenging one and further studies are required to formulate a complete theoretical model with therapeutic applications.

\section{CONCLUSIONS}

The specific ordered structure of amyloids renders them resistant to natural degradation mechanisms. A thorough study of the reasons and mechanisms behind the formation of such structures is a necessary step towards understanding the phenomenon of amyloidogenesis.

In order to analyze the formation of amyloids and amyloid-like aggregates using EM we have applied specific amyloid ligands, consisting of CR and TY molecules tagged with silver ions, as a contrast medium. CR and TY are capable of associating with each other and, together, form complexes with light chain aggregates (although, under favorable conditions, each dye remains capable of complexation on its own). Unlike CR, TY can bind silver ions strongly enough to survive complexation with proteins. CR serves as a carrier, enhancing the penetration capabilities of TY.

Preliminary studies using the above mentioned technique lead to the conclusion that aggregation of the $\mathrm{L}$ chains ( $\mathrm{IgG})$ is strongly mediated by the edge loop of the $\mathrm{V}$ domain (60-75 aa). This loop can form intermolecular $\beta$-bridges by association with its counterpart from the $\mathrm{V}$ domain of the adjacent $\mathrm{L}$ chain, producing threadlike aggregates, or participate in swapping, which leads to rigid higher-order structures. The resultant domains may, under favorable conditions, be fully packed and become impenetrable to $\mathrm{CR}$, much like native domains.

According to our research, supramolecular dyes bind to aggregates either by occupying sites which have been previously vacated by dislocated peptide chain fragments (in unstable domains), or adhere as supramolecular ribbon-like micellar forms to ordered fibrillary amyloid structures.

The distribution of silver-derived stains in amyloid structures produced by light chains and/or amyloid- $\beta$ 
peptides indicates their general tendency to form twisting chainlike structures.

\section{Acknowledgements}

This work was financially supported by the Jagiellonian University Medical College grant no. K/ZDS/001531.

The authors would like to acknowledge financial support from the Interdisciplinary PhD Studies "Molecular sciences for medicine" project (co-financed by the European Social Fund within the Human Capital Operational Programme).

Many thanks are due to Piotr Nowakowski, Anna Śmietanska and Romuald Bolesławski for technical and editorial support.

\section{REFERENCES}

Banach M, Konieczny L, Roterman I (2014) The fuzzy oil drop model, based on hydrophobicity density distribution, generalizes the influence of water environment on protein structure and function. I Theor Biol 359: 6-17. doi: 10.1016/j.jtbi.2014.05.007.

Barrow CJ, Yasuda A, Kenny PTM, Zagorski MG (1992) Solution conformations and aggregational properties of synthetic amyloid betapeptides of Alzheimer's disease. Analysis of circular dichroism spectra. J Mol Biol 225: 1075-1093. PMID: 1613791

Bennett MJ, Schlunegger MP, Eisenberg D (1995) 3D domain swapping: a mechanism for oligomer assembly. Protein Sci 4: 2455-24568. doi: 10.1002 /pro. 5560041202

Bross P, Corydon TJ, Andresen BS, Jørgensen MM, Bolund L, Gregersen N (1999) Protein misfolding and degradation in genetic diseases Hum Mutat 14: 186-198. doi: 10.1002/(SICI)10981004(1999)14:3<186::AID-HUMU2>3.0.CO;2-J

Chłopaś K, Jagusiak A, Konieczny L, Piekarska B, Roterman I, Rybarska J, Stopa B, Zemanem G, Bielańska E, Piwowar P, Sadlik K (2015) The use of Titan yellow dye as a metal ion binding marker for studies on the formation of specific complexes by supramolecular Congo red. Bio-Algorithms and Med-Systems 11: 9-18. doi: 10.1515/ bams-2015-0001

Churukian CJ (2000) Improved Puchtler's Congo red method for demonstrating amyloid. J Histotechnology 23: 139-141

Cohen FE. (1999) Protein misfolding and prion diseases. I Mol Biol 293: 313-320. PMID: 10550211

Colon W, Kelly JW (1992) Partial denaturation of transthyretin is sufficient for amyloid fibril formation in vitro. Biochemistry 31: 8654-8660. PMID: 1390650

Dealwis C, Wall J (2004) Towards understanding the structure-function relationship of human amyloid disease. Curr Drug Targets 5: 159-171. PMID: 15011949

Demeule B, Gurny R, Arvinte T (2007) Detection and characterization of protein aggregates by fluorescence microscopy. Int J Pharm 329: 37-45. PMID: 17005340

Elhaddaoui A, Pigorsch E, Delacourte A, Turrell S (1995) Competition of Congo red and Thioflavin S binding to amyloid sites in Alzheimer's diseased tissue. Biospectroscopy 1: 351-356

Ewert AS, Honegger A, Plückthun A (2004) Stability improvement of antibodies for extracellular and intracellular applications: CDR grafting to stable frameworks and structure-based framework engineering. Methods 34: 184-199. doi: 10.1016/j.ymeth.2004.04.007

Fan ZC, Shan L, Guddat LW, He XM, Gray WR, Raison RL, Edmundson AB (1992) Three-dimensional structure of an Fv from a human IgM immunoglobulin. J Mol Biol 228: 188-207. PMID: 1447781

Fink AL (1998) Protein aggregation: folding aggregates, inclusion bodies and amyloid. Fold Des 3: R9-R23. PMID: 9502314

Frid P, Anisimov SV, Popovic N (2007) Congo red and protein aggregation in neurodegenerative diseases. Brain Res Rev 53: 135-160. doi:10.1016/j.brainresrev.2006.08.001

Heegaard NH, Sen JW, Nissen MH (2000) Congophilicity (Congo red affinity) of different beta2-microglobulin conformations characterized by dye affinity capillary electrophoresis. J Chromatogr A 894: 319-327. PMID: 11100875

Helms LR, Wetzel R (1996) Specificity of abnormal assembly in immunoglobulin light chain deposition disease and amyloidosis $J \mathrm{Mol} \mathrm{Biol}$ 257: 77-86. PMID: 8632461

Howie AJ, Brewer DB (2009) Optical properties of amyloid stained by Congo red: history and mechanisms. Micron 40: 285-301. doi:10.1016/j.micron.2008.10.002

Hurle, MR, Helms LR, Li L, Chan W, Wetzel R (1994) A role for destabilizing amino acid replacements in light-chain amyloidosis. Proc Natl Acad Sci U S A 91: 5446-5450. PMID: 8202506
Iconomidou VA, Chryssikos GD, Gionis V, Hoenger A, Hamodrakas SJ (2003) FT-Raman spectroscopy as diagnostic tool of Congo red binding to amyloids. Biopolymers 72: 185-192. doi: 10.1002/bip.10344

Inouye H, Kirschner DA (2000) A beta fibrillogenesis: kinetic parameters for fibril formation from congo red binding. I Struct Biol 130: 123-129. PMID: 10940220

Jagusiak A, Konieczny L, Krol M, Marszalek P, Piekarska B, Piwowar P, Roterman I, Rybarska J, Stopa B, Zemanem G (2014) Intramolecular immunological signal hypothesis revived - structural background of signalling revealed by using Congo Red as a specific tool. Mini Rev Med Chem 14: 1104-1113. PMID: 25429660

Jiang X, Smith CS, Petrassi HM, Hammarström P, White JT, Sacchettini JC, Kelly JW (2001) An engineered transthyretin monomer that is nonamyloidogenic, unless it is partially denatured Biochemistry 40: 11442-11452. PMID: 11560492

Jin L-W, Claborn KA, Kurimoto M, Geday MA, Maezawa I, Sohraby F, Estrada M, Kaminsky W, Kahr B (2003) Imaging linear birefringence and dichroism in cerebral amyloid pathologies. Proc Natl Acad Sci U S A 100: 15294-15298. doi: 10.1073/pnas.2534647100

Kelly JW (1997) Amyloid fibril formation and protein misassembly: a structural quest for insights into amyloid and prion diseases. Structure 5: 595-600. PMID: 9195890

Khurana R, Uversky VN, Nielsen L, Fink AL (2001) Is Congo red an amyloid-specific dye? J Biol Chem 276: 22715-22721. PMID: 11410601

Khurana R, Gillespie JR, Talapatra A, Minert LJ, Ionescu-Zanetti C, Millett I, Fink AL (2001) Partially folded intermediates as critical precursors of light chain amyloid fibrils and amorphous aggregates. Biochemistry 40: 3525-3535. PMID: 11297418

Kim YS, Randolph TW, Manning MC, Stevens FJ, Carpenter JF (2003) Congo red populates partially unfolded states of an amyloidogenic protein to enhance aggregation and amyloid fibril formation $J$ Biol Chem 278: 10842-10850

King HGC, Pruden G (1967) The component of commercial titan yellow most reactive towards magnesium: Its isolation and use in determining magnesium in silicate minerals. Analyst 92: 83-90. doi: 10.1074/jbc.M212540200

Klunk WE, Jacob RF, Mason RP (1999) Quantifying amyloid betapeptide (Abeta) aggregation using the Congo red-Abeta (CR-abeta) spectrophotometric assay. Anal Biochem 266: 66-76. PMID: 9887214

Król M, Roterman I, Piekarska B, Konieczny L, Rybarska J, Stopa B, Spólnik P, Szneler E (2005) An approach to understand the complexation of supramolecular dye Congo red with immunoglobulin L chain lambda. Biopolymers 77: 155-162. doi: 10.1002/bip.20197

Lakdawala AS, Morgan DM, Liotta DC, Lynn DG, Snyder JP (2002) Dynamics and fluidity of amyloid fibrils: a model of fibrous protein aggregates. J Am Chem Soc 124: 15150-15151. PMID: 12487571

Lendel C, Bolognesi B, Wahlström A, Dobson CM, Gräslund A (2010) Detergent-like interaction of Congo red with the amyloid beta peptide. Biochemistry 49: 1358-1360. doi: 10.1021/bi902005t

Liu Y, Hart PJ, Schlunegger MP, Eisenberg D (1998) The crystal structure of a 3D domain-swapped dimer of RNase A at a 2.1-A resolution. Proc Natl Acad Sci US A 95: 3437-3442. PMID: 9520384

Lu JX, Qiang W, Yau WM, Schwieters CD, Meredith SC, Tycko R (2013) Molecular structure of $\beta$-amyloid fibrils in Alzheimer's disease brain tissue. Cell 154: 1257-1268. doi: 10.1016/j.cell.2013.08.035

Lührs T, Ritter C, Adrian M, Riek-Loher D, Bohrmann B, Döbeli H, Schubert D, Riek R (2005) 3D structure of Alzheimer's amyloidbeta(1-42) fibrils. Proc Natl Acad Sci U S A 102: 17342-17347. doi: $10.1073 /$ pnas.0506723102

Maji SK, Wang L, Greenwald J, Riek R (2009) Structure-activity relationship of amyloid fibrils. FEBS Lett 583: 2610-2617. doi: 10.1016/j.febslet.2009.07.003

Mizobata T, Kawata Y (1994) The guanidine-induced conformational changes of the chaperonin GroEL from Escherichia coli. Evidence for the existence of an unfolding intermediate state. Biochim Biophys Acta 1209: 83-88. PMID: 7947986

Nagradova NK (2002) Three-dimensional domain swapping in homooligomeric proteins and its functional significance. Biochem (Moscow) 67: 839-849. PMID: 12223084

Nelson R, Esenberg D (2006) Recent atomic models of amyloid fibril structure. Curr Op Struct Biol 16: 260-265. PMID: 16563741

Pedersen M.Ø., Mikkelsen K, Behrens MA, Pedersen JS, Enghild JJ, Skrydstrup T, Malmendal A, Nielsen NC (2010) NMR reveals two-step association of Congo Red to amyloid $\beta$ in low-molecularweight aggregates. J Phys Chem B 114: 16003-16010

Pellarin R, Schuetz P, Guarnera E, Caflisch AJ (2010) Amyloid fibril polymorphism is under kinetic control. I Am Chem Soc 132: 1496014970. doi: 10.1021/ja106044u

Petkova AT, Yau WM, Tycko R (2006) Experimental constraints on quaternary structure in Alzheimer's beta-amyloid fibrils. Biochemistry 45: 498-512. doi: 10.1021/bi051952q

Picken MM (2001) The changing concepts of amyloid. Arch Patol Lab Med 125: 38-43. doi: 10.1043/0003-9985(2001)125<0038:TCCOA> 2.0.CO;2 
Piekarska B, Konieczny L, Rybarska J, Stopa B, Zemanek G, Szeler E, Król M, Nowak M, Roterman I (2001) Heat-induced formation of a specific binding site for self-assembled Congo Red in the $\mathrm{V}$ domain of immunoglobulin L chain lambda. Biopolymers 59: 446-456. doi: 10.1002/1097-0282(200111)59:6<446::AID-BIP1049>3.0.CO;2-X

Pollack SJ, Sadler IIJ, Hawtin SR, Tailor VJ, Shearman MS (1995) Sulfonated dyes attenuate the toxic effects of beta-amyloid in a structure-specific fashion. Neurosci Lett 197: 211-214. PMID: 8552301

del Pozo YL, Ortiz E, Sánchez R, Sánchez-López R, Güereca L, Murphy CL, Allen A, Wall JS, Fernández-Velasco DA, Solomon A, Becerril B (2008) Influence of the germline sequence on the thermodynamic stability and fibrillogenicity of human lambda 6 light chains. Proteins 72: 684-692. doi: 10.1002/prot.21934

Pratim Bose P, Chatterjee U, Xie L, Johansson J, Göthelid E, Arvidsson PI (2010) Effects of Congo red on aß(1-40) fibril formation process and morphology. ACS Chem Neurosci 1: 315-324. doi: $10.1021 / \operatorname{cn} 900041 \mathrm{x}$

Qin Z, Hu D, Zhu M, Fink AL (2007) Structural characterization of the partially folded intermediates of an immunoglobulin light chain leading to amyloid fibrillation and amorphous aggregation. Biochemistry 46: 3521-3531. doi: 10.1021/bi061716v

Quintas A, Vaz DC, Cardoso I, Saraiva MJ, Brito RM (2001) Tetramer dissociation and monomer partial unfolding precedes protofibril formation in amyloidogenic transthyretin variants. J Biol Chem 276: 27207-27213. doi: 10.1074/jbc.M101024200

Roterman I, Rybarska J, Konieczny L, Skowronek M, Stopa B, Piekarska B, Bakalarski $G$ (1998) Congo red bound to $\alpha$-1-proteinase inhibitor as a model of supramolecular ligand and protein complex Computers Chem 22: 61-70

Schormann N, Murrell JR, Liepnieks JJ, Benson MD (1995) Tertiary structure of an amyloid immunoglobulin light chain protein: a proposed model for amyloid fibril formation. Proc Natl Acad Sci US A 92: 9490-9494. PMID: 7568160

Serpell LC (2000) Alzheimer's amyloid fibrils: structure and assembly. Biochim Biophys Acta 1502: 16-30. PMID: 10899428

Sinha N, Tsai CJ, Nussinov R (2001) A proposed structural model for amyloid fibril elongation: domain swapping forms an interdigitating beta-structure polymer. Protein Eng 14: 93-103. PMID: 11297667

Skowronek M, Stopa B, Konieczny L, Rybarska J, Szneler E, Bakalarski G, Roterman I (1998) Self- assembly of Congo red - A theoreti$\mathrm{cal}$ and experimental approach to identify its supramolecular organization in water and salt solution. Biolpolymers 46: 267-281.

Spólnik P, Konieczny L, Piekarska B, Rybarska J, Stopa B, Zemanem G, Król M, Roterman I (2004) Instability of monoclonal myeloma protein may be identified as susceptibility to penetration and bind- ing by newly synthesized Congo red derivatives. Biochimie 86: $397-$ 401. PMID: 15358056

Stopa B, Górny M, Konieczny L, Piekarska B, Rybarska J, Skowronek M, Roterman I (1998) Supramolecular ligands: monomer structure and protein ligation capability. Biochimie 80: 963-968. PMID: 9924974

Stopa B, Piekarska B, Konieczny L, Rybarska J, Spólnik P, Zemanem G, Roterman I, Król M (2003) The structure and protein binding of amyloid-specific dye reagents. Acta Bioch Pol 50: 1213-1227. PMID: 14740008

Teng PK, Anderson NJ, Goldschmidt L, Sawaya MR, Sambashivan S, Eisenberg D (2012) Ribonuclease A suggests how proteins selfchaperone against amyloid fiber formation. Protein Sci 21: 26-37. doi: 10.1002 /pro. 754

Turnell WG, Finch JT (1992) Binding of the dye congo red to the amyloid protein pig insulin reveals a novel homology amongst amyloid-forming peptide sequences. J Mol Biol 227: 1205-1223. PMID: 1433294

Tycko R (2004) Progress towards a molecular-level structural understanding of amyloid fibrils. Curr Opin Struct Biol 14: 96-103. doi: 10.1016/j.sbi.2003.12.002

Wall J, Schell M, Murphy C, Hrncic RR, Stevens FJ, Solomon A (1999) Thermodynamic instability of human lambda 6 light chains: correlation with fibrillogenicity. Biochemistry 38: 14101-14108. PMID: 10529258

Wood SJ, Maleeff B, Hart T, Wetzel R (1996) Physical, morphological and functional differences between ph 5.8 and 7.4 aggregates of the Alzheimer's amyloid peptide Abeta. J Mol Biol 256: 870-877. doi: 10.1006/imbi.1996.0133

Woodcock S, Henrissat B, Sugiyama J (1995) Docking of congo red to the surface of crystalline cellulose using molecular mechanics. Biopolymers 36: 201-210. doi: 10.1002/bip.360360208

Wu C, Wang Z, Lei H, Zhang W, Duan Y (2007) Dual binding modes of Congo red to amyloid protofibril surface observed in molecular dynamics simulations. J Am Chem Soc 129: 1225-1232. doi: 10.1021/ ja0662772

Wu C, Scott J, Shea JE (2012) Binding of Congo red to amyloid protofibrils of the Alzheimer $\mathrm{A} \beta(9-40)$ peptide probed by molecular dynamics simulations. Biophys J 103: 550-557. doi: 10.1016/j. bpj.2012.07.008

Yang M, Lei M, Huo S (2003) Why is Leu55-->Pro55 transthyretin variant the most amyloidogenic: insights from molecular dynamics simulations of transthyretin monomers. Prot Sci 12: 1222-1231. doi: $10.1110 /$ ps.0239703 